\title{
Quality of acute myocardial infarction care in England and Wales during the COVID-19 pandemic: linked nationwide cohort study
}

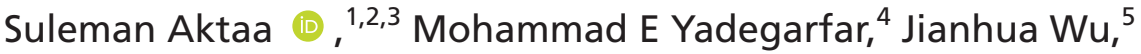 \\ Muhammad Rashid, ${ }^{6}$ Mark de Belder, ${ }^{7}$ John Deanfield, ${ }^{8}$ \\ Francois Schiele (D) , 9 Mark Minchin, ${ }^{10}$ Mamas Mamas, ${ }^{11}$ Chris P Gale ${ }^{1,2,3}$
}

- Additional supplemental material is published online only. To view, please visit the journal online (http://dx.doi. org/10.1136/bmjqs-2021013040).

For numbered affiliations see end of article.

\section{Correspondence to} Dr Suleman Aktaa, Leeds Institute for Data Analytics and Leeds Institute of Cardiovascular and Metabolic Medicine, University of Leeds, Leeds LS2 9JT, UK; s.aktaa@leeds.ac.uk

Received 14 January 2021 Accepted 10 June 2021 Published Online First 22 June 2021

\begin{abstract}
Background and objective The impact of the COVID-19 pandemic on the quality of care for patients with acute myocardial infarction (AMI) is uncertain. We aimed to compare quality of AMI care in England and Wales during and before the COVID-19 pandemic using the 2020 European Society of Cardiology Association for Acute Cardiovascular Care quality indicators (QIs) for AMI. Methods Cohort study of linked data from the AMI and the percutaneous coronary intervention registries in England and Wales between 1 January 2017 and 27 May 2020 (representing 236743 patients from 186 hospitals). At the patient level, the likelihood of attainment for each QI compared with pre COVID-19 was calculated using logistic regression. The date of the first national lockdown in England and Wales (23 March 2020) was chosen for time series comparisons.

Results There were 10749 admissions with AMI after 23 March 2020. Compared with before the lockdown, patients admitted with AMI during the first wave had similar age (mean 68.0 vs 69.0 years), with no major differences in baseline characteristics (history of diabetes ( $25 \%$ vs $26 \%$ ), renal failure (6.4\% vs $6.9 \%$ ), heart failure ( $5.8 \%$ vs $6.4 \%$ ) and previous myocardial infarction $(22.9 \%$ vs $23.7 \%))$, and less frequently had high Global Registry of Acute Coronary Events risk scores (43.6\% vs $48.6 \%)$. There was an improvement in attainment for 10 $(62.5 \%)$ of the 16 measured QIs including a composite QI $(43.8 \%$ to $45.2 \%$, OR $1.06,95 \% \mathrm{Cl} 1.02$ to 1.10$)$ during, compared with before, the lockdown. Conclusion During the first wave of the COVID-19 pandemic in England and Wales, quality of care for AMl as measured against international standards did not worsen, but improved modestly.
\end{abstract}

\section{INTRODUCTION}

The COVID-19 pandemic has impacted on the structure and organisation of services delivered through the National Health Service (NHS) with knock-on effects on the management of a number of acute cardiovascular conditions including acute myocardial infarction (AMI) in the UK. ${ }^{1-4}$ For patients admitted to hospital with AMI, guideline-indicated therapies such as invasive coronary angiography, timely reperfusion and secondary prevention medications improve survival, ${ }^{5}$ and professional organisations in the UK recommended the perpetuation of these therapies during the pandemic. ${ }^{67}$ Yet, an earlier study found an increase in 30-day mortality and a reduction in the proportion of invasive coronary angiography during the national lockdown for patients with non-ST segment elevation myocardial infarction (NSTEMI). ${ }^{8}$ There has been, however, no comprehensive evaluation of the quality of AMI care during the first national lockdown and no study has used recognised standards for such an investigation.

Quality indicators (QIs) have been increasingly used as a mechanism to measure broad aspects of care, ${ }^{9}$ identify unwanted variation $^{1011}$ and drive quality improvement. ${ }^{12}$ For AMI, a suite of QIs exist which are valid, ${ }^{13}$ internationally recognised ${ }^{14}$ and have built on earlier indicators that have an inverse association with mortality. ${ }^{15-19}$ We used the UK national cardiovascular registries to investigate the quality of AMI care according to these indicators during the first national lockdown in the COVID-19 pandemic. This may help understand changes in the processes of AMI care during the time of national crisis and identify areas for improvement.

\section{METHODS}

\section{Data and population}

We used linked data from the UK national AMI and percutaneous coronary intervention (PCI) registries, namely the Myocardial Ischaemia National Audit Project $(\text { MINAP) })^{20}$ and the National Audit 
of Percutaneous Coronary Intervention (NAPCI), championed by the British Cardiovascular Intervention Society. ${ }^{22}$ MINAP and NAPCI registries have been described previously. ${ }^{20} 23$ The National Institute for Cardiovascular Outcomes Research (NICOR), commissioned through the Healthcare Quality Improvement Partnership, manages MINAP, NAPCI and other registries.

NICOR has support under section 251 of the NHS Act 2006 (Ref: NIGB: ECC 1-06 (d)/2011) to use patient information for medical research without consent. Thus, ethical approval was not required under NHS research governance arrangements. We conducted our study in compliance with the Declaration of Helsinki using the MINAP and NAPCI databases.

\section{Sample selection}

We included all adult patients ( $\geqslant 18$ years of age), discharged alive with ST-segment elevation myocardial infarction (STEMI) or NSTEMI from MINAP between 1 January 2017 and 27 May 2020. Data related to PCI were obtained from the NAPCI registry using each patient's unique NHS number to deterministically link patients between the two registries. Where multiple admissions for the same patient were recorded, the earlier admission was used to reduce potential bias from previous treatments. Patients with no valid NHS number were excluded.

\section{Quality indicators}

We used the 2020 European Society of Cardiology (ESC) Association for Acute Cardiovascular Care (ACVC) QIs for AMI, which comprise 26 indicators. The eligibility criteria for each QI was determined according to the specifications provided in the ESC ACVC document. ${ }^{14}$

\section{Outcomes}

The outcome was quality of AMI care. Care quality was quantified according to the degree to which eligible patients received the care outlined in the QIs prior to, compared with after, 23 March 2020 (up to 27 May 2020). This date was chosen for the time series comparison because it corresponded with the first national lockdown in England and Wales.

\section{Statistical analysis}

Patient baseline characteristics, comorbidities and treatments were reported according to the study period and type of AMI as percentages and numbers for categorical variables, means and SDs for parametric continuous variables, and medians and IQRs for non-parametric variables. Baseline differences between each diagnosis were tested using $\chi^{2}$ test for categorical variables, $t$-test for continuous parametric and the Mann-Whitney U test for non-parametric variables. At the patient level, the likelihood of attainment for each QI compared with that before the COVID-19 pandemic was estimated using logistic regression.
All analyses were performed on complete cases. All tests were two-sided, and statistical significance was considered as $\mathrm{p}$ value $<0.05$. Statistical analyses were performed in Stata IC V.14.2 and R V.3.4.3.

\section{RESULTS}

\section{Study population}

Data for 236743 patients admitted with AMI to one of 186 NHS hospitals were included. Of those, 152 109 (64.3\%) patients had NSTEMI, and the median age was $69.0(58-79)$ years with 75918 (32.2\%) patients being women. The cohort following lockdown (10 749) were compared with the period chosen before lockdown (225 994). Table 1 shows the demographics, comorbidities, in-hospital treatment and discharge details according to the study period. Data are presented according to the type of AMI in online supplemental table 1. Compared with before the lockdown, patients admitted with AMI during the first wave had similar age (mean 68.0 vs 69.0 years), similar baseline characteristics (history of diabetes ( $25 \%$ vs $26 \%$ ), renal failure $(6.4 \%$ vs $6.9 \%)$, heart failure $(5.8 \%$ vs $6.4 \%)$ and previous myocardial infarction $(22.9 \%$ vs 23.7\%)) and less frequently had high Global Registry of Acute Coronary Events (GRACE) risk scores (43.6\% vs $48.6 \%$ ) (table 1 ).

\section{Quality of care assessment}

Data from the national registries enabled the direct measurement of $16(61.5 \%)$ of the 26 ESC ACVC QIs. The QIs that could not be assessed included the planned duration of dual anti-platelet therapy, the QIs within the patient satisfaction domain and the objective risk-stratification using validated tools. GRACE risk scores, however, were indirectly derived for 193 177 (81.6\%) patients. In addition, while participating in a network for STEMI management, taking part in a registry and routine monitoring to reperfusion times in STEMI could not be directly measured, these form part of routine practice in the UK. The outcome QI (30-day mortality) may be obtained from data linkage with the Civil Registration of Deaths Register, but was not evaluated for this work because mortality had been previously investigated ${ }^{8}$ and this study concerned processes of care.

\section{Quality of care during the COVID-19 pandemic}

During, compared with before, the national lockdown, in England and Wales there was an improvement in attainment for 10 (62.5\%) QIs, with evidence for a slight reduction in attainment for the other QIs that could be measured using the datasets (table 2). Figure 1 shows the OR for QI attainment during the lockdown referenced to the pre-COVID period.

Overall, there was a slight increase in attainment for the composite QI after the first national lockdown $(43.8 \%$ to $45.2 \%$, OR $1.06,95 \% \mathrm{CI}$ 1.02 to 1.10$)$ suggesting good overall adherence to 


\section{Original research}

Table 1 Baseline characteristics for admissions with AMI, by study period

\begin{tabular}{|c|c|c|c|c|}
\hline & Before lockdown & Since lockdown & All & Missing data $\%(\mathrm{n})$ \\
\hline Patients, $\mathrm{n}$ & 225994 & 10749 & 236743 & \\
\hline Hospitals, n & 186 & 186 & 186 & \\
\hline \multicolumn{5}{|l|}{ Demographics } \\
\hline Female, \% (n) & $32.2(72667)$ & $30.3(3251)$ & $32.2(75918)$ & $0.3(621)$ \\
\hline Age (years), median (IQR) & $69.0(58-79)$ & $68.0(58-77)$ & $69.0(58-79)$ & $0(0)$ \\
\hline \multicolumn{5}{|l|}{ Baseline characteristics } \\
\hline Heart rate at hospitalisation (bpm), median (IQR) & $77(66-90)$ & $77(66-90)$ & $77(66-90)$ & $3.4(7960)$ \\
\hline Systolic blood pressure (mm Hg), median (IQR) & $137(120-156)$ & $140(121-160)$ & $137(120-157)$ & $3.3(7826)$ \\
\hline Initial creatinine, $\mu$ mol/L, median (IQR) & $85(71-105)$ & $83(70-101)$ & $85(71-104)$ & $4.6(10824)$ \\
\hline \multicolumn{5}{|l|}{ Diagnosis } \\
\hline STEMI & $35.7(80564)$ & $37.9(4070)$ & $35.8(84634)$ & $0(0)$ \\
\hline NSTEMI & $64.4(145430)$ & $62.1(6679)$ & $64.3(152$ 109) & $0(0)$ \\
\hline \multicolumn{5}{|l|}{ GRACE } \\
\hline Score, median (IQR) & $121(96-151)$ & $117(95-143)$ & $121(96-151)$ & $18.4(43566)$ \\
\hline Low, \% (n) & $19.1(35628)$ & $21.4(1863)$ & $19.4(37491)$ & \\
\hline Intermediate, \% (n) & 32.1 (59 278) & $35.0(3049)$ & $32.3(62327)$ & \\
\hline High, \% (n) & $48.6(89563)$ & $43.6(3796)$ & $48.3(93359)$ & \\
\hline \multicolumn{5}{|l|}{ Killip class } \\
\hline I, \% (n) & $82.3(166682)$ & $85.5(8263)$ & $82.4(174945)$ & $10.4(24511)$ \\
\hline$\|, \%(n)$ & $11.4(23106)$ & $9.3(895)$ & $11.3(24001)$ & \\
\hline III, \% (n) & $4.6(9320)$ & $3.6(348)$ & $4.6(9668)$ & \\
\hline IV, \% (n) & $1.7(3459)$ & $1.7(159)$ & $1.7(3618)$ & \\
\hline \multicolumn{5}{|l|}{ Comorbidities } \\
\hline Diabetes, \% (n) & $26.0(58767)$ & $25.0(2685)$ & $26.0(61452)$ & $0(0)$ \\
\hline $\mathrm{COPD}, \%(n)$ & $14.8(33539)$ & $15.6(1568)$ & $14.8(35$ 107) & $0(0)$ \\
\hline Chronic heart failure, \% (n) & $6.4(14453)$ & $5.8(623)$ & $6.4(15076)$ & $0(0)$ \\
\hline Chronic renal failure, \% (n) & $6.9(15646)$ & $6.4(684)$ & $6.9(16330)$ & $0(0)$ \\
\hline Cerebrovascular disease, \% (n) & $7.3(16436)$ & $6.5(696)$ & $7.2(17$ 132) & $0(0)$ \\
\hline Peripheral vascular disease, \% (n) & 4.0 (9109) & $3.8(409)$ & $4.0(9518)$ & $0(0)$ \\
\hline Hypertension, \% (n) & 47.6 (107 532) & $46.7(5016)$ & $47.5(112548)$ & $0(0)$ \\
\hline Previous MI, \% (n) & $23.7(47647)$ & $22.9(2145)$ & $23.7(49792)$ & $11.3(26692)$ \\
\hline Previous angina, \% (n) & 20.7 (40 919) & $18.3(1683)$ & $20.6(42602)$ & $12.7(30083)$ \\
\hline Previous PCI, \% (n) & $15.4(30483)$ & $16.3(1499)$ & $15.5(31982)$ & $12.6(29742)$ \\
\hline Previous CABG, \% (n) & $7.2(14324)$ & $6.6(610)$ & $7.2(14934)$ & $12.5(29565)$ \\
\hline \multicolumn{5}{|l|}{ In-hospital procedures } \\
\hline Invasive coronary angiography, \% (n) & $71.3(160795)$ & $73.1(7841)$ & $71.3(168636)$ & $0.15(354)$ \\
\hline $\mathrm{PCl}, \%(n)$ & $51.4(116202)$ & $56.7(6094)$ & $51.7(122296)$ & $0(0)$ \\
\hline CABG, \% (n) & $2.0(4610)$ & $0.9(96)$ & $2.0(4706)$ & $0(0)$ \\
\hline \multicolumn{5}{|l|}{ Medications at discharge } \\
\hline Aspirin, \% (n) & 97.9 (167 286) & $98.0(7994)$ & $97.9(175280)$ & $24.4(57682)$ \\
\hline $\mathrm{P} \mathrm{Y}_{12}$ inhibitor, \% (n) & $96.6(165723)$ & $97.7(8062)$ & $96.6(173785)$ & $24.0(56849)$ \\
\hline Beta blocker, \% (n) & $96.1(156943)$ & $96.5(7557)$ & $96.1(164500)$ & $27.7(65590)$ \\
\hline ACEi or ARB, \% (n) & $94.2(148284)$ & $94.5(7275)$ & $94.2(155559)$ & $30.3(71643)$ \\
\hline Statins, \% (n) & $97.3(168402)$ & $97.6(8118)$ & $97.3(176520)$ & $23.5(55639)$ \\
\hline \multicolumn{5}{|l|}{ Lifestyle advice } \\
\hline Cardiac rehabilitation, \% (n) & 88.8 (159 999) & $88.4(7652)$ & $88.8(167651)$ & $20.2(47903)$ \\
\hline Smoking cessation advice, \% (n) & $74.6(48821)$ & $78.1(2320)$ & $74.6(51$ 141) & 71.1 (168 331) \\
\hline Dietary advice, \% (n) & 89.8 (148 959) & $91.1(7067)$ & 89.8 (156 026) & $26.6(63013)$ \\
\hline
\end{tabular}

ACEi, angiotensin converting enzyme inhibitor; ARB, angiotensin receptor blocker; bpm, beats per minute; CABG, coronary artery bypass graft; COPD, chronic obstructive pulmonary disease; GRACE, Global Registry of Acute Coronary Events; MI, myocardial infarction; NSTEMI, non-ST segment elevation myocardial infarction; PCI, percutaneous coronary intervention; STEMI, ST-segment elevation myocardial infarction. 
Table 2 Quality indicator attainment before and during the COVID-19 pandemic for admissions with AMI

\begin{tabular}{|c|c|c|c|c|}
\hline QI (receipt or availability) & Before lockdown ( $n=225994)$ & After lockdown ( $n=10749)$ & All $(n=236743)$ & $P$ value \\
\hline Hospital use of hs-cTn for NSTEMI, \% (n) & $70.4(99983)$ & $82.1(5403)$ & $70.9(105386)$ & $<0.001$ \\
\hline $\begin{array}{l}\text { Pre-hospital interpretation of ECG for STEMI, \% } \\
\text { (n) }\end{array}$ & 76.6 (55 274) & $80.7(2696)$ & $76.7(57970)$ & $<0.001$ \\
\hline Reperfusion among eligible for STEMI, \% (n) & $80.6(64931)$ & $80.3(3268)$ & 80.6 (68 199) & $<0.001$ \\
\hline Timely reperfusion for STEMI, \% (n) & $80.1(52$ 024) & $79.8(2608)$ & $54632(80.10)$ & $<0.001$ \\
\hline $\begin{array}{l}\text { Invasive coronary angiography within } 24 \text { hours } \\
\text { for NSTEMI, \% (n) }\end{array}$ & $35.1(24208)$ & $49.1(1811)$ & $35.8(26019)$ & $<0.001$ \\
\hline Radial access for invasive procedures, \% (n) & $87.6(87880)$ & $91.4(3924)$ & $87.8(91804)$ & $<0.001$ \\
\hline $\begin{array}{l}\text { Median time (min) from ECG to arterial access* } \\
\text { for STEMI, median (IQR) }\end{array}$ & $87.4(65.5-118.0)$ & $91.8(70.0-131.1)$ & $87.4(65.5-120.1)$ & 0.017 \\
\hline LVEF assessment before hospital discharge, \% (n) & $62.3(140848)$ & $61.6(6621)$ & $62.3(147469)$ & $<0.001$ \\
\hline In-hospital measurement of LDL-C,$+ \%$ (n) & $56.0(126619)$ & $61.5(6613)$ & $56.3(133232)$ & $<0.001$ \\
\hline Adequate $\mathrm{P}_{2} \mathrm{Y}_{12}$ inhibition on discharge, $\%$ (n) & 96.6 (165 723) & $97.7(8062)$ & 96.6 (173 785) & $<0.001$ \\
\hline Parenteral anticoagulation, \% (n) & $82.9(147369)$ & $82.6(6564)$ & $82.9(153933)$ & $<0.001$ \\
\hline Dual antiplatelet therapy on discharge, \% (n) & 95.5 (156 034) & $96.7(7624)$ & 95.5 (163 658) & $<0.001$ \\
\hline High-intensity statin on discharge $\neq, \%$ (n) & $97.3(168402)$ & $97.6(8118)$ & $97.3(176520)$ & $<0.001$ \\
\hline ACEi for patients with reduced LVEF, \% (n) & 96.7 (45 542) & $96.5(2179)$ & $96.7(47721)$ & $<0.001$ \\
\hline $\begin{array}{l}\text { Beta blockers for patients with reduced LVEF, \% } \\
\text { (n) }\end{array}$ & $98.1(48352)$ & $98.3(2260)$ & $98.1(50612)$ & $<0.001$ \\
\hline Composite all-or-none, \% (n) & $43.8(98968)$ & $45.2(4863)$ & $43.9(103831)$ & $<0.001$ \\
\hline \multicolumn{5}{|c|}{$\begin{array}{l}\text { *Door to balloon time. } \\
\text { †Serum cholesterol. } \\
\text { ‡Discharged on statin. } \\
\text { ACEi, angiotensin converting enzyme inhibitor; AMI, acute myocardial infarction; hscTn, high-sensitivity cardiac troponin; LDL-C, low-density lipoprotein } \\
\text { cholesterol; LVEF, left ventricular ejection fraction; NSTEMI, non-ST segment elevation myocardial infarction; QI, quality indicator; STEMI, ST-segment } \\
\text { elevation myocardial infarction. }\end{array}$} \\
\hline
\end{tabular}

guidelines-indicated therapies for AMI during the COVID-19 pandemic.

\section{DISCUSSION}

This real-world naturalistic study evaluated the quality of AMI care in England and Wales before and during the COVID-19 pandemic using routinely collected nationwide registry data. We found that the NHS provided high-quality AMI care during the pandemic as measured against international standards. In particular, we found that early detection and timely invasive investigation for NSTEMI were delivered at much higher rates, while STEMI reperfusion was slightly delayed than prior to the UK lockdown. Such insights were gained by means of routinely collected cardiovascular data. These findings highlight the role that the UK national cardiovascular registries may play in the evaluation of processes of AMI care in times of need.

Others have described changes in the patterns of treatment for patients with AMI during the COVID-19 pandemic, ${ }^{1-38}$ but no study has quantified the breadth or depth of AMI care on a national level using validated QIs. Similar findings of an overall improvement in the quality of care have recently been reported for patients with stroke in the UK. ${ }^{24}$ Taken together, this emphasises the consequences of a national crisis on the delivery of processes of care for acute cardiovascular conditions and may help identify areas for improvement.

One may only speculate as to the reasons for improved care quality for AMI following the national lockdown. Given that there was a reported decline of between $16 \%$ and $40 \%$ in admissions with AMI to hospitals following the first UK lockdown, the modest improvement in attainment of the majority of the QIs during the pandemic could be explained by a relative increase in availability of cardiology staff and resources. ${ }^{12825}$ That is, a reduction in admissions for AMI, with the maintenance of a specialist emergency heart attack service, would provide greater opportunities for specialist staff to deliver higher quality care. ${ }^{3}$ Indeed, at the time, the British Cardiovascular Society recommended the UK national heart attack service to continue as previously and not to revert to historical treatments for AMI such as thrombolysis. ${ }^{67}$ This was in contrast to recommendations during the early stages of the pandemic to adopt a 'thrombolysis-first' approach. ${ }^{26}$ Given the decline in admissions with AMI, our findings suggest that care quality could be further improved with appropriate staffing and resources.

However, it is possible that other factors were at play. This includes the preparedness of dedicated services (and with this additional staff availability and attention) and the prioritisation of hospital discharges 


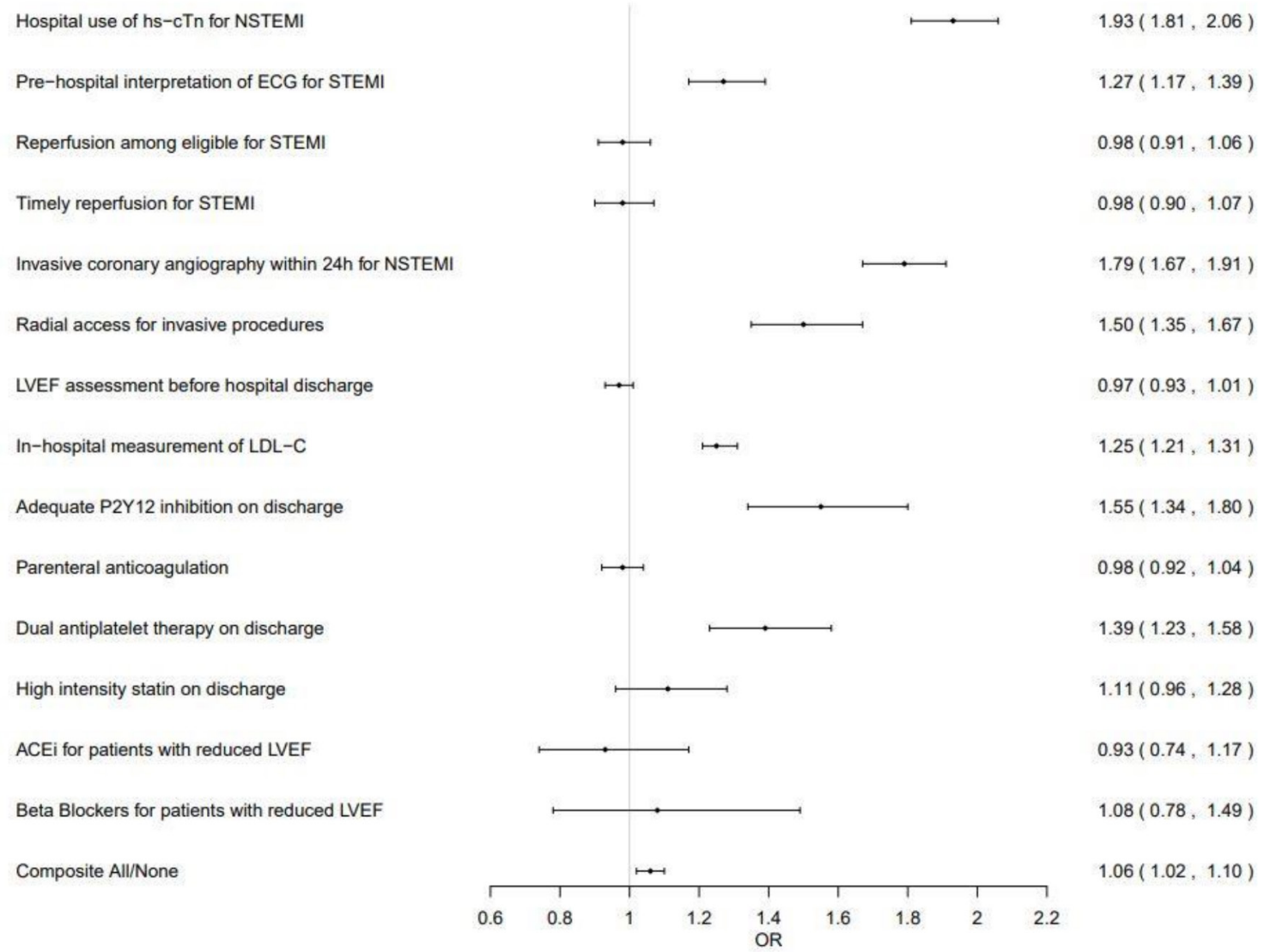

Figure 1 Quality indicator attainment for patients with AMI during the first UK national lockdown compared with the pre-COVID-19 period. ACEi, ACE inhibitor; AMI, acute myocardial infarction; DAPT, dual anti-platelet therapy; hs-cTn, high-sensitivity cardiac troponin; ICA, invasive coronary angiography; LDL-C, low-density lipoprotein cholesterol; LVEF, left ventricular ejection fraction; NSTEMI, non-ST elevation myocardial infarction; STEMI, ST elevation myocardial infarction. Balloon inflation time was substituted for arterial access time, serum cholesterol for LDL cholesterol, and statin for high intensity statin.

(and therefore greater attention to the provision of care prior to leaving hospital). ${ }^{27}$ Moreover, the 'shut down' of normal elective activity, ${ }^{28}{ }^{29}$ which spanned all services, would have enabled the NHS to be better equipped to receive and treat patients with AMI. It is also plausible that the recording of data into the national registries was more selective, with a bias towards patients who were lower risk, had better care and who were more likely to be discharged alive (previous work has suggested that missing data is associated with 30-day mortality for STEMI and NSTEMI). ${ }^{30}$

The delay in STEMI reperfusion observed in our study is consistent with other $\mathrm{UK}^{3}$ and international ${ }^{31}$ studies, and may be related to the changes to STEMI service during the pandemic including the redeployment of catheter laboratory staff to other intensive care environments. ${ }^{27}$ Furthermore, the slight reduction in the assessment of left ventricular ejection fraction prior to hospital discharge and the prescription of angiotensin converting enzyme inhibitors or angiotensin receptor blockers for those with a reduced ejection fraction, as well as the increase in radial access use after the lockdown, may be due to the fact that there was an imperative to make available hospital beds and therefore enable the early hospital discharge of stable patients following AMI.

Our study does emphasise an opportunity to integrate local efforts with those wider afield that aim to evaluate and improve the quality of AMI care. The ESC QIs have been designed to enable the assessment of care quality for AMI, according to international clinical practice guidelines. ${ }^{32}$ Equally, MINAP and NAPCI are used as tools for audit and evaluation of NHS heart attack services. ${ }^{33}$ Hitherto, we were only able to measure $61.5 \%$ of the ESC AMI QIs against these two national registers. We propose that routine national data collection aligns to and harmonises with national and international standards for the measurement of quality of care. ${ }^{34}$ Equally, we recognise that while information such as health-related quality of life may be difficult to capture via national registries, ${ }^{35}$ greater alliance may help enhance the comprehensiveness of data collection systems in the UK. ${ }^{36} 37$

Our study has limitations. MINAP does not collect information pertaining to all admissions with AMI 
across the NHS. ${ }^{38}$ It is possible that care quality for those admissions recorded were systematically different from those not in the registry. Nonetheless, MINAP does collect detailed clinical information pertaining to the majority of admissions in England and Wales with AMI, and is the largest single healthcare system AMI registry. ${ }^{20}$ We substituted statin therapy for high-intensity statin, serum cholesterol for lowdensity lipoprotein cholesterol, and balloon inflation time for arterial access time. While these are slightly different aspects of care to the ones proposed in the ESC ACVC QIs, they provide insights into current practice of pharmacotherapy following AMI. This was a retrospective cohort study which has bias inherent to its observational design.

\section{CONCLUSION}

The COVID-19 pandemic created a natural experiment for the NHS. During this period, quality of care for AMI as measured against international standards did not worsen, but improved modestly. Give the decline in admissions with AMI, our findings could suggest that care quality may be further improved with appropriate staffing and resources. Implicit in the study is the notion that routinely collected data in concert with standardised measures of care quality allow appropriate evaluation of care quality.

\footnotetext{
Author affiliations

${ }^{1}$ Leeds Institute for Data analytics, University of Leeds, Leeds, UK

${ }^{2}$ Department of Cardiology, Leeds Teaching Hospitals NHS Trust, Leeds, UK

${ }^{3}$ Leeds Institute of Cardiovascular and Metabolic Medicine, University of Leeds, Leeds, UK

${ }^{4}$ School of Population Health and Environmental Sciences, King's College London, London, UK

${ }^{5}$ Division of Clinical and Translational Research, School of Dentistry, University of Leeds, Leeds, UK

${ }^{6}$ Keele Cardiovascular Research Group, Centre for Prognosis Research, Institutes of Applied Clinical Science and Primary Care and Health Sciences, Keele University, Keele, UK

${ }^{7}$ National Institute for Cardiovascular Outcomes Research, Barts Health NHS Trust, London, UK

${ }^{8}$ Institute of Cardiovascular Sciences, University College London, London, UK

${ }^{9}$ Cardiology, University Hospital J Minjoz, Besancon, France

${ }^{10} \mathrm{Health}$ and Social Care Directorate, NICE, Manchester, UK

${ }^{11}$ Institute for Science \& Technology in Medicine, Keele University, Keele, UK
}

\section{Twitter Suleman Aktaa@SulemanAktaa}

Contributors SA, MEY and CPG were responsible for the study design and concept. JW and MR performed the data cleaning and data analysis. SA, MEY and CPG wrote the first draft of the manuscript and all authors participated in the writing of the paper.

Funding The authors have not declared a specific grant for this research from any funding agency in the public, commercial or not-for-profit sectors.

Competing interests None declared.

Patient consent for publication Not required.

Provenance and peer review Not commissioned; externally peer reviewed.

Data availability statement Data may be obtained from a third party on ethical approval and are not publicly available.
All data relevant to the study are included in the article or uploaded as online supplemental information.

Supplemental material This content has been supplied by the author(s). It has not been vetted by BMJ Publishing Group Limited (BMJ) and may not have been peer-reviewed. Any opinions or recommendations discussed are solely those of the author(s) and are not endorsed by BMJ. BMJ disclaims all liability and responsibility arising from any reliance placed on the content. Where the content includes any translated material, BMJ does not warrant the accuracy and reliability of the translations (including but not limited to local regulations, clinical guidelines, terminology, drug names and drug dosages), and is not responsible for any error and/or omissions arising from translation and adaptation or otherwise.

This article is made freely available for use in accordance with BMJ's website terms and conditions for the duration of the covid-19 pandemic or until otherwise determined by BMJ. You may use, download and print the article for any lawful, noncommercial purpose (including text and data mining) provided that all copyright notices and trade marks are retained.

\section{ORCID iDs}

Suleman Aktaa http://orcid.org/0000-0002-9854-481X Francois Schiele http://orcid.org/0000-0003-4974-6551

\section{REFERENCES}

1 Mafham MM, Spata E, Goldacre R, et al. COVID-19 pandemic and admission rates for and management of acute coronary syndromes in England. Lancet 2020;396:381-9.

2 Pessoa-Amorim G, Camm CF, Gajendragadkar P, et al. Admission of patients with STEMI since the outbreak of the COVID-19 pandemic: a survey by the European Society of Cardiology. Eur Heart J Qual Care Clin Outcomes 2020;6:210-6.

3 Kwok CS, Gale CP, Kinnaird T, et al. Impact of COVID-19 on percutaneous coronary intervention for ST-elevation myocardial infarction. Heart 2020;106:1805-11.

4 Rashid Hons M, Gale Hons CP, Curzen Hons N, et al. Impact of coronavirus disease 2019 pandemic on the incidence and management of out-of-hospital cardiac arrest in patients presenting with acute myocardial infarction in England. J Am Heart Assoc 2020;9:e018379.

5 Collet J-P, Thiele H, Barbato E, et al. 2020 ESC guidelines for the management of acute coronary syndromes in patients presenting without persistent ST-segment elevation. Eur Heart J 2021;42:1289-367.

6 Curzen N. An extended statement by the British Cardiovascular Intervention Society president regarding the COVID-19 pandemic. Interv Cardiol 2020;15:e01.

7 Speciality guides for patient management during the coronavirus pandemic. Clinical guide for the management of cardiology patients during the coronavirus pandemic. Available: https://www.nice.org.uk/Media/Default/About/ COVID-19/Specialty-guides/specialty-guide-cardiolgycoronavirus.pdf

8 Wu J, Mamas M, Rashid M, et al. Patient response, treatments, and mortality for acute myocardial infarction during the COVID-19 pandemic. Eur Heart J Qual Care Clin Outcomes 2021;7:238-46.

9 Duclos A, Chollet F, Pascal L, et al. Effect of monitoring surgical outcomes using control charts to reduce major adverse events in patients: cluster randomised trial. BMJ 2020;371:m3840.

10 Hofstede SN, Ceyisakar IE, Lingsma HF, et al. Ranking hospitals: do we gain reliability by using composite rather than individual indicators? BMJ Qual Saf 2019;28:94-102. 
11 Mulley AG. Improving productivity in the NHS. BMJ 2010;341:c3965.

12 Friebel R, Steventon A. Composite measures of healthcare quality: sensible in theory, problematic in practice. BMJ Qual Saf 2019;28:85-8.

13 Schiele F, Gale CP, Simon T, et al. The 2020 ESC-ACVC quality indicators for the management of acute myocardial infarction applied to the FAST-MI registries. Eur Heart J Acute Cardiovasc Care 2021;10:207-15.

14 Schiele F, Aktaa S, Rossello X, et al. 2020 Update of the quality indicators for acute myocardial infarction: a position paper of the Association for Acute Cardiovascular Care: the study group for quality indicators from the ACVC and the NSTE-ACS guideline group. Eur Heart J Acute Cardiovasc Care 2021;10:224-33.

15 Schiele F, Gale CP, Bonnefoy E, et al. Quality indicators for acute myocardial infarction: a position paper of the Acute Cardiovascular Care Association. Eur Heart J Acute Cardiovasc Care 2017;6:34-59.

16 Zusman O, Bebb O, Hall M, et al. International comparison of acute myocardial infarction care and outcomes using quality indicators. Heart 2019;105:820-5.

17 Rossello X, Medina J, Pocock S, et al. Assessment of quality indicators for acute myocardial infarction management in 28 countries and use of composite quality indicators for benchmarking. Eur Heart J Acute Cardiovasc Care 2020;9:911-22.

18 Schiele F, Gale CP, Simon T, et al. Assessment of quality indicators for acute myocardial infarction in the FAST-MI (French registry of acute ST-elevation or non-ST-elevation myocardial infarction) registries. Circ Cardiovasc Qual Outcomes 2017;10.

19 Bebb O, Hall M, Fox KAA, et al. Performance of hospitals according to the ESC ACCA quality indicators and 30-day mortality for acute myocardial infarction: national cohort study using the United Kingdom Myocardial Ischaemia National Audit Project (MINAP) register. Eur Heart J 2017;38:974-82.

20 Wilkinson C, Weston C, Timmis A, et al. Cohort profile: the Myocardial Ischaemia National Audit Project (MINAP). European Heart Journal - Quality of Care and Clinical Outcomes 2019;39:19-22.

21 Simms AD, Weston CF, West RM, et al. Mortality and missed opportunities along the pathway of care for ST-elevation myocardial infarction: a national cohort study. Eur Heart J Acute Cardiovasc Care 2015;4:241-53.

22 Ludman PF, British Cardiovascular Intervention Society. British Cardiovascular Intervention Society Registry for audit and quality assessment of percutaneous coronary interventions in the United Kingdom. Heart 2011;97:1293-7.

23 Ludman P. British Cardiovascular Intervention Society database: insights into interventional cardiology in the United Kingdom. Heart 2019;105:1289.
24 Douiri A, Muruet W, Bhalla A, et al. Stroke care in the United Kingdom during the COVID-19 pandemic. Stroke 2021;52:2125-33.

25 Wu J, Mamas MA, Mohamed MO, et al. Place and causes of acute cardiovascular mortality during the COVID-19 pandemic. Heart 2021;107:113-9.

26 Zhang L, Fan Y, Lu Z. Experiences and lesson strategies for cardiology from the COVID-19 outbreak in Wuhan, China, by 'on the scene' cardiologists. Eur Heart J 2020;41:1788-90.

27 Kwok CS, Gale CP, Curzen N, et al. Impact of the COVID-19 pandemic on percutaneous coronary intervention in England: insights from the British Cardiovascular Intervention Society PCI database cohort. Circ Cardiovasc Interv 2020;13:e009654.

28 Iacobucci G. Covid-19: all non-urgent elective surgery is suspended for at least three months in England. BMJ 2020;368:m1106.

29 Morris EJA, Goldacre R, Spata E, et al. Impact of the COVID-19 pandemic on the detection and management of colorectal cancer in England: a population-based study. Lancet Gastroenterol Hepatol 2021;6:199-208.

30 Gale CP, Cattle BA, Moore J, et al. Impact of missing data on standardised mortality ratios for acute myocardial infarction: evidence from the myocardial ischaemia national audit project (MINAP) 2004-7. Heart 2011;97:1926-31.

31 Rattka M, Dreyhaupt J, Winsauer C, et al. Effect of the COVID-19 pandemic on mortality of patients with STEMI: a systematic review and meta-analysis. Heart 2021;107:482-7.

32 Aktaa S, Batra G, Wallentin L. European Society of Cardiology methodology for the development of quality indicators for the quantification of cardiovascular care and outcomes. Eur Heart J Qual Care Clin Outcomes 2020;388.

33 Ludman P. British Cardiovascular Intervention Society database: insights into interventional cardiology in the United Kingdom. Heart 2019;105:1289-89.

34 Wallentin L, Gale CP, Maggioni A, et al. EuroHeart: European Unified Registries On Heart Care Evaluation and Randomized Trials. Eur Heart J 2019;40:2745-9.

35 Bhatt DL, Drozda JP, Shahian DM, et al. ACC/AHA/STS statement on the future of registries and the performance measurement enterprise. J Am Coll Cardiol 2015;66:2230-45.

36 Elshehaly M, Randell R, Brehmer M. QualDash: Adaptable Generation of Visualisation Dashboards for Healthcare Quality Improvement. IEEE Trans Vis Comput Graph 2020;PP(99).

37 Alabas OA, West RM, Gillott RG, et al. Evaluation of the Methods and Management of Acute Coronary Events (EMMACE)-3: protocol for a longitudinal study. BMJ Open 2015;5:e006256.

38 Herrett E, Shah AD, Boggon R, et al. Completeness and diagnostic validity of recording acute myocardial infarction events in primary care, hospital care, disease registry, and national mortality records: cohort study. BMJ 2013;346:f2350. 